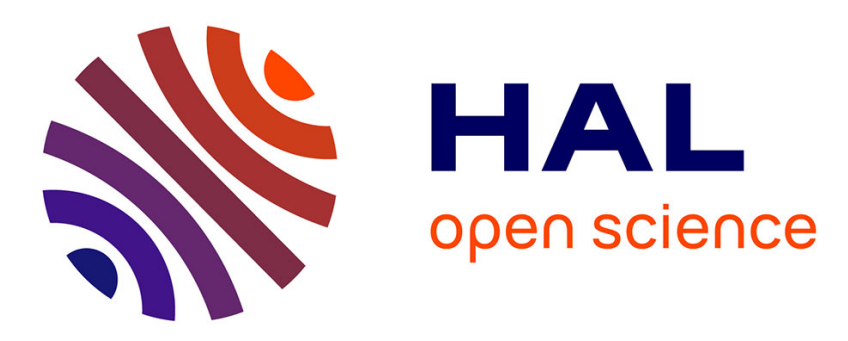

\title{
Heterogeneity in susceptibility to infection can explain high reinfection rates
}

\author{
Paula Rodrigues, M. Gabriela M. Gomes, Alessandro Margheri, Carlota \\ Rebelo
}

\section{> To cite this version:}

Paula Rodrigues, M. Gabriela M. Gomes, Alessandro Margheri, Carlota Rebelo. Heterogeneity in susceptibility to infection can explain high reinfection rates. Journal of Theoretical Biology, 2009, 259 (2), pp.280. 10.1016/j.jtbi.2009.03.013 . hal-00554590

\section{HAL Id: hal-00554590 https://hal.science/hal-00554590}

Submitted on 11 Jan 2011

HAL is a multi-disciplinary open access archive for the deposit and dissemination of scientific research documents, whether they are published or not. The documents may come from teaching and research institutions in France or abroad, or from public or private research centers.
L'archive ouverte pluridisciplinaire HAL, est destinée au dépôt et à la diffusion de documents scientifiques de niveau recherche, publiés ou non, émanant des établissements d'enseignement et de recherche français ou étrangers, des laboratoires publics ou privés. 


\section{Author's Accepted Manuscript}

Heterogeneity in susceptibility to infection can explain high reinfection rates

Paula Rodrigues, M. Gabriela M. Gomes, Alessandro Margheri, Carlota Rebelo
PII: S0022-5193(09)00126-X
DOI: doi:10.1016/j.jtbi.2009.03.013
Reference: YJTBI 5502

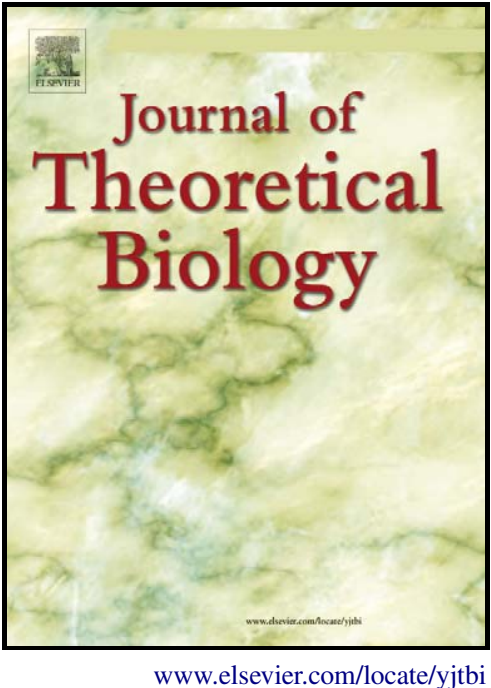

To appear in: $\quad$ Journal of Theoretical Biology

Received date: $\quad 31$ October 2008

Revised date: $\quad 16$ February 2009

Accepted date: 11 March 2009

Cite this article as: Paula Rodrigues, M. Gabriela M. Gomes, Alessandro Margheri and Carlota Rebelo, Heterogeneity in susceptibility to infection can explain high reinfection rates, Journal of Theoretical Biology (2009), doi:10.1016/j.jtbi.2009.03.013

This is a PDF file of an unedited manuscript that has been accepted for publication. As a service to our customers we are providing this early version of the manuscript. The manuscript will undergo copyediting, typesetting, and review of the resulting galley proof before it is published in its final citable form. Please note that during the production process errors may be discovered which could affect the content, and all legal disclaimers that apply to the journal pertain. 


\title{
Heterogeneity in susceptibility to infection can explain high reinfection rates
}

\author{
Paula Rodrigues ${ }^{a, c, *}$, M. Gabriela M. Gomes ${ }^{a, b}$, Alessandro Margheri ${ }^{b}$, Carlota \\ Rebelo $^{b}$ \\ ${ }^{a}$ Instituto Gulbenkian de Ciência, Apartado 14, 2781-901 Oeiras, Portugal. \\ ${ }^{b}$ Fac. Ciências de Lisboa and Centro de Matemática e Aplicações Fundamentais, Av. Prof. Gama Pinto \\ 2, 1649-003 Lisboa, Portugal. \\ ${ }^{c}$ Fac. Ciências e Tecnologia da Universidade Nova de Lisboa, Monte da Caparica, 2825-114 Caparica, \\ Portugal. \\ * Corresponding author. Instituto Gulbenkian de Ciência, Apartado 14, 2781-901 Oeiras, Portugal, \\ Fax:+351 214407970 . \\ E-mail addresses: pcpr@igc.gulbenkian.pt (P. Rodrigues); ggomes@igc.gulbenkian.pt (M.G.M. Gomes), \\ margheri@ptmat.fc.ul.pt (A. Margheri), carlota@ptmat.fc.ul.pt (C. Rebelo).
}

\begin{abstract}
Heterogeneity in susceptibility and infectivity is inherent to infectious disease transmission in nature. Here we are concerned with the formulation of mathematical models that capture the essence of heterogeneity while keeping a simple structure suitable of analytical treatment. We explore the consequences of host heterogeneity in the susceptibility to infection for epidemiological models for which immunity conferred by infection is partially protective, known as Susceptible-Infected-Recovered-Infected (SIRI) models. We analyze the impact of heterogeneity on disease prevalence and contrast the susceptibility profiles of the subpopulations at risk for primary infection and reinfection. We present a systematic study in the case of two frailty groups.

We predict that the average rate of reinfection may be higher than the average rate of primary infection, which may seem paradoxical given that primary infection induces lifelong partial protection. Infection generates a selection mechanism whereby fit individuals remain in $S$ and frail individuals are transferred to $R$. If this effect is strong enough
\end{abstract}


we have a scenario where, on average, the rate of reinfection is higher than the rate of primary infection even though each individual has a risk reduction following primary infection. This mechanism may explain high rates of tuberculosis reinfection recently reported.

Finally, the enhanced benefits of vaccination strategies that target the high-risk groups are quantified.

Keywords: partial immunity, interventions, tuberculosis. 


\section{Introduction}

Heterogeneity in susceptibility and infectivity is an important feature of many infectious diseases and has been considered to improve the accuracy of epidemiological models. In the analysis of these models, focus has been on the impact of heterogeneity in the final size of epidemics (Ball, 1985; Miller, 2007) and on its consequences to disease control (Anderson \& Britton, 1998; Britton, 1998) and data interpretation (Gart, 1968; Anderson \& May, 1991). In the context of SIR epidemic models, it has been shown that the final size of the epidemic is reduced when the risk of infection is heterogeneously distributed in the population, both for the deterministic and the stochastic formulations (Gart, 1968; Ball, 1985; Anderson \& Britton, 1998). More recently, results were extended to the investigation of epidemic spread on a random network (Miller, 2007).

In this work we explore the consequences of host heterogeneity in the susceptibility to infection for endemic models for which immunity conferred by infection is not fully protective, known as Susceptible-Infected-Recovered-Infected (SIRI) models. The model is expanded to accommodate multiple frailty groups classified accordingly to risk of infection. We are concerned not only with the impact on disease prevalence but also on how transmission changes the risk profile of the population groups that are subject to reinfection. The SIRI model exhibits two important thresholds in transmission: the endemic threshold that marks the transmission intensity necessary to maintain disease endemic in a population; and the reinfection threshold that indicates whether self-sustained transmission occurs in a population which has developed a degree of partial immunity (Gomes et al., 2004). The reinfection threshold separates two fundamentally distinct model behaviors. Low endemic levels with SIR-like transmission are maintained below threshold, while high endemic levels with SISlike transmission characterise the regime above threshold. Therefore, first we consider the case of SIR and SIS models, exploring their simplicity and mathematical tractability to extract general trends. We describe how disease prevalence, risk profiles for specific population compartments, and contribution of the high-risk group to overall incidence, change with the parameters describing heterogeneity. Second, the same framework is used to explore the SIRI 
model. Of particular interest is the interplay between reinfection and the risk profile for the uninfected compartments, $S$ and $R$.

The results offer a plausible explanation for observations of higher than expected reinfection rates. In particular, rates of reinfection that surpass rates of first infection have been reported for tuberculosis in a high transmission setting in South Africa (Verver et al., 2005). Naively, one could attribute this effect to some form of immunologically dependent enhancement whereby immunological memory would render individuals more susceptible to subsequent infections. An alternative hypothesis suggested by the analysis presented here is that relatively high rates of reinfection can result from the presence of a high-risk group that, being at higher frequency in the recovered compartment due to selection imposed by the first infection, can sustain rates of reinfection that are, on average, higher than the rates of first infection even in the presence of partially protective immunity.

Heterogeneity has many implications for public health policy. In particular, we characterise how the impact of vaccination strategies varies with transmission intensity, and quantify the benefit of targeting high-risk groups.

\section{The model}

To incorporate heterogeneity in the infection risk in an SIRI transmission model we use a formulation close to the one presented by Ball (1985) for SIR epidemic models. We assume that the population is divided in $n$ different subgroups according to the susceptibility to infection, $\alpha_{i}$. These groups will be referred as frailty groups (Coutinho et al., 1999). Within each frailty group, individuals are classified according to their disease history into susceptible, infectious or recovered. A schematic version of the model is shown in Figure 1.

Figure 1: about here

It is assumed that the $n$ frailty groups have constant size over time and that they represent different proportions of the total population, $\gamma_{i}$, where $\sum_{i=1}^{n} \gamma_{i}=1$. Individuals are born into each group at the rate $\mu \gamma_{i}$. We use $S_{i}, I_{i}$ and $R_{i}$ as the proportion of each class from the 
frailty group $i$ in the total population. Hence we have $\sum_{i=1}^{n}\left(S_{i}+I_{i}+R_{i}\right)=1$. In the following we denote by $I$ the proportion of infectious in the population, that is $I=\sum_{i=1}^{n} I_{i}$.

For concreteness, we fix the parameters as described in table 1. The table describes an

Table 1: about here

average life expectancy of 70 years (that is $\mu=1 / 70$ ) and an average infectious period of one week (that is $\tau=52$ ). The factor reducing the risk of infection as a result of acquired immunity is $\sigma=0.25$. For the limiting cases of the SIR and SIS models, parameter $\sigma$ is 0 or 1 , respectively. Parameters $\beta, \gamma_{i}$ and $\alpha_{i}$ are varied to explore different scenarios for transmission intensity and host heterogeneity. Each frailty group has an average risk of infection that differs from the population average by a factor $\alpha_{i}$, which we refer to as the relative risk of infection (Gart, 1968; Ball, 1985). We assume that this factor controls the rate of infection and reinfection in the $i$ th frailty group. In general, the parameters are chosen to resemble an acute respiratory infection in a developed country. However, we stress that the results are valid for a wider set of parameters. Differences reside more on the quantitative than on the qualitative behavior. The model can be written as a system of $3 n$ differential equations

$$
\left\{\begin{aligned}
S_{i}^{\prime} & =\mu \gamma_{i}-\lambda \alpha_{i} S_{i}-\mu S_{i} \\
I_{i}^{\prime} & =\lambda \alpha_{i} S_{i}+\sigma \lambda \alpha_{i} R_{i}-(\tau+\mu) I_{i} \\
R_{i}^{\prime} & =\tau I_{i}-\sigma \lambda \alpha_{i} R_{i}-\mu R_{i}, \quad i=1, \ldots, n
\end{aligned}\right.
$$

where $\lambda=\beta I$. To ensure comparison between different assumptions on risk distribution, including the comparison with the homogeneous version of the model, we impose the normalization $\bar{\alpha}=\sum \alpha_{i} \gamma_{i}=1$.

Throughout this paper we analyse the case $n=2$. We denote by $\gamma$ the proportion of individuals belonging to the low-risk group (that is, $\gamma_{1}=\gamma$ and $\gamma_{2}=1-\gamma$ ). For a given population structure $(\gamma)$ we vary the infection risk distribution by changing $\alpha_{1}$, obtaining $\alpha_{2}$ through the normalization $\alpha_{1} \gamma+\alpha_{2}(1-\gamma)=1$. We use the variance as a summary measure 
of variations on $\alpha_{1}$,

$$
\operatorname{var}_{\alpha}=\left(\bar{\alpha}-\alpha_{1}\right)^{2} \gamma+\left(\bar{\alpha}-\alpha_{2}\right)^{2}(1-\gamma)=\frac{\left(1-\alpha_{1}\right)^{2} \gamma}{1-\gamma}
$$

Note that for a given population structure the variance is a decreasing function of $\alpha_{1}$. The homogeneous model is obtained for $\alpha_{1}=\bar{\alpha}=1$ which, consistently, corresponds to zero variance.

\subsection{Basic reproduction number}

The basic reproduction number is an important concept in the study of epidemiological models. We recall that in the case of the corresponding model for homogeneous populations $\left(\alpha_{1}=1\right)$ the basic reproduction number is given by

$$
R_{0}=\frac{\beta}{\tau+\mu}
$$

Considering the heterogeneous model, the basic reproduction number is not altered. In fact,

$$
R_{0}^{H E T}=\frac{\beta}{\tau+\mu} \sum_{i=1}^{2} \alpha_{i} \gamma_{i}=\frac{\beta \bar{\alpha}}{\tau+\mu}=\frac{\beta}{\tau+\mu}=R_{0} .
$$

For a more detailed discussion on the calculation of the basic reproduction number in heterogeneous populations see Hyman \& Li (2000) or Diekmann et al. (1990). A threshold condition for endemicity is given by $R_{0}=1$ (the disease dies out if $R_{0}<1$ and becomes endemic if $\left.R_{0}>1\right)$.

Note that the basic reproduction number for the entire population is a weighted average of the basic reproduction number within each independent frailty group, $R_{0 i}$, given by

$$
R_{0}^{H E T}=\sum_{i} \frac{\alpha_{i} \beta}{\tau+\mu} \gamma_{i}=\sum_{i} R_{0 i} \gamma_{i}
$$

Therefore, if the basic reproduction number for each group is greater than one, then the disease is also endemic in the entire population. On the other hand, it is not necessary to have 
all reproductive numbers greater than one to have endemicity.

\section{The limit cases, $\operatorname{SIR}(\sigma=0)$ and $\operatorname{SIS}(\sigma=1)$}

Before studying the SIRI model, we analyze the impact of host heterogeneity in the case of SIR and SIS models, corresponding to $\sigma=0$ and $\sigma=1$, respectively. The identification between the SIS model and our model with $\sigma=1$ is made in a natural way, by collapsing the classes $S$ and $R$ of this last model into a class $S+R$, which we identify with the susceptible class of the SIS model. However, in order to make possible the comparison between the limit case with $\sigma=1$ and the intermediate SIRI model, in what follows we keep distinct the $S$ and $R$ classes even for $\sigma=1$. Actually, we will consider the class $S+R$ in Remark 3.1, where we examine the effect of heterogeneity on the prevalence in the SIS framework. All results stated in this section are proved in the appendix.

\subsection{Endemic equilibrium}

For $\sigma=0$ or 1 , system (2.1) has one disease-free equilibrium of the form $E_{0}^{\sigma}=(\gamma, 1-$ $\gamma, 0,0,0,0)$. Above $R_{0}=1$, the system has also an endemic equilibrium, $E_{1}^{\sigma}$. Stability results for these equilibria are stated in the two theorems below. We use the superscript $\sigma$ to denote the correspondence with the $\operatorname{SIR}(\sigma=0)$ or the SIS $(\sigma=1)$ models.

Theorem 3.1.1. For $\sigma=0$ or 1 , the disease-free equilibrium, $E_{0}^{\sigma}$, of system (2.1) is globally asymptotically stable if $R_{0}<1$ and it is unstable for $R_{0}>1$.

Theorem 3.1.2. Let $\sigma=0$ or 1 and assume that $R_{0}>1$. Then system (2.1) has exactly one endemic equilibrium, $E_{1}^{\sigma}$, that is globally asymptotically stable.

Figure 2: about here

We analyze the impact of heterogeneity on disease prevalence at equilibrium. Figure 2 illustrates how disease prevalence changes for different assumptions on population structure 
and distribution of infection risk for the SIR and SIS models. We observe that for a fixed $R_{0}$, the equilibrium disease prevalence is lower when assuming heterogeneous populations. From each plot, it is evident that for fixed $\gamma$, the prevalence curve goes down as variance increases. Comparing the three plots it is also apparent that for fixed $\alpha_{1}$, the prevalence curve goes down as the proportion of the population at low risk $(\gamma)$ increases. Moreover, the disease prevalence appears to increase monotonically with the transmission potential, $R_{0}$. The following theorem summarizes these results.

Theorem 3.1.3. Let $\sigma=0$ or $\sigma=1$ and let $I^{\sigma}, \sigma=0,1$, designate the disease prevalence at equilibrium, for the corresponding system (2.1) with $R_{0}>1$. Then, for $\gamma$ and $\alpha_{1} \in(0,1)$

$$
\begin{aligned}
\frac{\partial I^{\sigma}}{\partial \gamma} \leq 0, \quad & \frac{\partial I^{\sigma}}{\partial \alpha_{1}} \geq 0 \\
\text { and } \quad & \frac{\partial I^{\sigma}}{\partial R_{0}}>0, \quad \sigma=0,1 .
\end{aligned}
$$

Previous studies based on the SIR framework have shown that heterogeneity in susceptibility to infection gives rise to smaller epidemics (Gart, 1968; Ball, 1985; Anderson \& Britton, 1998). Here we find that disease prevalence at equilibrium is also lower in the presence of heterogeneity, and this is true for both SIR and SIS models. This effect is more pronounced the higher the variance in risk distribution.

\subsection{Infection risk profiles}

The profiles of the infection risk, within the susceptible and the recovered classes at endemic equilibrium depend on assumptions on population heterogeneity and transmission intensity. For $\sigma=0,1$, we define the average risk factor among susceptible and recovered individuals as

$$
\bar{\alpha}_{S}^{\sigma}=\frac{\alpha_{1} S_{1}^{*}+\alpha_{2} S_{2}^{*}}{S_{1}^{*}+S_{2}^{*}}, \quad \bar{\alpha}_{R}^{\sigma}=\frac{\alpha_{1} R_{1}^{*}+\alpha_{2} R_{2}^{*}}{R_{1}^{*}+R_{2}^{*}}
$$

where $S_{i}^{*}$ and $R_{i}^{*}$ are the susceptible and recovered individuals in each frailty group, represented as proportions of the total population at endemic equilibrium. Figure 3 shows contour plots for the average risk factor among individuals never infected $(S)$ and those infected and 
Figure 3: about here

recovered at least once $(R)$. Note, however, that these factors are further multiplied by $\lambda$ and $\sigma \lambda$ to produce the average per capita rates of infection in $S$ and $R$, respectively. This figure reflects how selection imposed by infection acts on the risk profiles.

In the SIR model, the average risk decreases as $R_{0}$ increases both for never-infected individuals and previously-infected individuals (Figure 3(a) and Figure 3(b), respectively). This selection mechanism underlies counter-intuitive trends that will emerge with the exploration of $\sigma \in(0,1)$ in section 4 , such as rates of reinfection decreasing with increasing $R_{0}$ and rates of reinfection appearing higher than rates of first infection even in the presence of partially protective immunity.

In the SIS model, selection maintains a large proportion of the high-risk group in the infected class and the mechanism is not entirely visible in the uninfected sub-population. Note that the average risk among never-infected individuals is roughly constant with $R_{0}$ (Figure $3(\mathrm{c})$ ) while among previously-infected individuals (Figure 3(d)) the average risk decreases with increasing $R_{0}$ as in the susceptible class of the SIR model (Figure 3(a)).

The properties observed for $\bar{\alpha}_{S}^{0}$ are summarized in the following theorem, proved in the appendix.

Theorem 3.2.1. Let $R_{0}>1$. Then, for $\gamma$ and $\alpha_{1} \in(0,1)$

$$
\begin{gathered}
\frac{\partial \bar{\alpha}_{S}^{0}}{\partial \gamma} \leq 0, \quad \frac{\partial \bar{\alpha}_{S}^{0}}{\partial \alpha_{1}} \geq 0 \\
\text { and } \quad \frac{\partial \bar{\alpha}_{S}^{0}}{\partial R_{0}}<0
\end{gathered}
$$

The decrease on the average risk of infection of the susceptible class explains how prevalence decreases with population heterogeneity. In fact, the average force of infection, $\bar{\lambda}^{0}$, depends on the transmission intensity and on the average infection risk of the population 
subject to infection,

$$
\bar{\lambda}^{0}=\lambda^{0} \bar{\alpha}_{S}^{0}=\beta I^{0} \bar{\alpha}_{S}^{0}=\mu\left(R_{0} \bar{\alpha}_{S}^{0}-1\right),
$$

where we expressed $I^{0}$ as a function of $\bar{\alpha}_{S}^{0}$ according to formula (B.2) given in section B of the appendix. Directly from Theorem 3.2.1 it follows that heterogeneity decreases the force of infection, since

$$
\frac{\partial \bar{\lambda}^{0}}{\partial \gamma} \leq 0, \quad \frac{\partial \bar{\lambda}^{0}}{\partial \alpha_{1}} \geq 0
$$

Remark 3.1. As mentioned above, when $\sigma=1$ we can identify our model with a SIS model through the identification of the class $S+R$ with the susceptible class of the SIS. It is then natural to investigate the effect of heterogeneity on prevalence by considering the dependence on the parameters of the average risk of infection of the $S+R$ class, $\bar{\alpha}_{S+R}^{1}$, defined as

$$
\bar{\alpha}_{S+R}^{1}:=\frac{\alpha_{1}\left(S_{1}^{*}+R_{1}^{*}\right)+\alpha_{2}\left(S_{2}^{*}+R_{2}^{*}\right)}{\left(S_{1}^{*}+R_{1}^{*}\right)+\left(S_{2}^{*}+R_{2}^{*}\right)}
$$

and of the corresponding force of infection, $\bar{\lambda}^{1}$, defined as $\bar{\lambda}^{1}=\lambda^{1} \bar{\alpha}_{S+R}^{1}=\beta I^{1} \bar{\alpha}_{S+R}^{1}$. This is easily done, since from Remark B.1 and equation (A.4) in the appendix we have, respectively, that $\bar{\alpha}_{S+R}^{1}=\bar{\alpha}_{S}^{0} \quad$ and that $\bar{\lambda}^{1}=\frac{\tau+\mu}{\mu} \bar{\lambda}^{0}$. As a consequence, $\bar{\lambda}^{1}$ satisfies the inequalities (3.7), and we conclude that the decrease on the average risk of infection of the susceptible plus recovered class explains how prevalence decreases with population heterogeneity in the SIS model.

Finally, as a side remark, we would like to note that with respect to the quantities defined in (3.3), it is $\bar{\alpha}_{S+R}^{1}=\frac{\bar{\alpha}_{S}^{1} S^{*}+\bar{\alpha}_{R}^{1} R^{*}}{S^{*}+R^{*}}$.

Despite having the same infectivity, the frailty groups contribute differently to the force of infection. Disease is more easily spread on the high-risk group due to its increased susceptibility, so the relative size of class $I_{2}$ is also greater. To further explore how the contribution of the high-risk group to the total proportion of infections changes with transmission intensity and heterogeneity, we define the quotient $Q^{\sigma}=I_{2}^{\sigma} / I^{\sigma}$ at equilibrium. For $\sigma=0$ or $\sigma=1$, 
the contribution of the high-risk group, $Q^{\sigma}$, decreases as transmissibility increases and it is greater when this frailty group is larger ( $\gamma$ close to 0$)$ or when its relative risk of infection is further from the population average ( $\alpha_{1}$ close to 0$)$. The following theorem summarizes these results.

Theorem 3.2.2. Let $R_{0}>1$. Then, for $\gamma$ and $\alpha_{1} \in(0,1)$

$$
\begin{array}{r}
\frac{\partial Q^{\sigma}}{\partial \gamma} \leq 0, \quad \frac{\partial Q^{\sigma}}{\partial \alpha_{1}} \leq 0 \\
\text { and } \quad \frac{\partial Q^{\sigma}}{\partial R_{0}}<0 \quad \sigma=0,1 .
\end{array}
$$

Overall, the contribution of the high-risk group can vary from $\alpha_{2}$ times its relative size, near the epidemic threshold, to its relative size, for sufficiently high transmission. This can have important consequences for the effectiveness of interventions, specially in low endemic regions where the groups with increased risk have more impact. We will focus more on this aspect when studying the SIRI model.

\section{The SIRI model}

\subsection{Endemic equilibrium}

Here we consider the effect of heterogeneity in the intermediate scenario where infection induces partial immunity. It is assumed that individuals are protected while infected but regain some susceptibility upon recovery. Susceptibility to reinfection is reduced by a factor $\sigma \in[0,1]$, compared to susceptibility to first infection. Endemic equilibria and infection risk profiles have been analyzed for the limiting cases $\sigma=0,1$ (corresponding to SIR, SIS models) in section 3. In both cases, disease persistence is determined by the threshold condition, $R_{0}>1$, irrespective of population structure, sustaining levels of infection that are generally much higher in the SIS scenario due to reinfection. In the intermediate case, another threshold has been identified, $R_{0}=1 / \sigma$ (Gomes et al., 2004, 2005), to describe a transition from SIRto SIS-like behavior. In section $\mathrm{C}$ of the appendix we show that the same expression holds 
for the reinfection threshold in the presence of heterogeneity in susceptibility to infection.

Quantitative discrepancies between epidemiological data and model results have been reported previously and generally attributed to case sub-notification or population heterogeneity not captured by simple models (Fine \& Clarkson (1982), Anderson \& May (1985), van Boven at al. (2001)). Systematic investigations of these factors are expected to provide valuable insights with wide application in infectious disease epidemiology. In section 3 we have shown that heterogeneity in susceptibility to infection reduces prevalence of infection in SIR and SIS models and here we extend this conclusion to the general SIRI framework. Figure 4 shows the

Figure 4: about here

endemic equilibrium for different infection risk profiles of the population. When heterogeneity is considered the disease prevalence is lower than in the homogeneous case, and this effect is more pronounced when the variance, $v a r_{\alpha}$, is higher (high $\gamma$ and low $\alpha_{1}$ ). These trends are observed for $0 \leq \sigma \leq 1$, including the particular cases $\sigma=0,1$, analyzed previously.

\subsection{Infection risk profiles}

The reduction in disease prevalence is associated with the changes in the infection risk profile imposed by transmission on both susceptible and recovered classes. In this section we analyze how the average infection risk of susceptible and recovered individuals change with $R_{0}$ and heterogeneity (here represented by the proportion the population with low risk, $\gamma$, and risk of these individuals relative to the average, $\left.\alpha_{1}\right)$. We remark that when $\sigma \in(0,1)$ the average risk factors in the susceptible and recovered classes, $\bar{\alpha}_{S}$ and $\bar{\alpha}_{R}$ respectively, are defined as in $(3.3)$.

Figure 5 illustrates the average risk factor for susceptible and recovered classes for $\gamma=0.8$ and $\sigma=0.25$, by means of contour plots in the parameter space of transmissibility, $R_{0}$, and heterogeneity, $\alpha_{1}$. Generally, the average risk among susceptible individuals decreases as $R_{0}$ increases (Figure 5(a)) while the opposite is observed among recovered individuals (Figure 5(b)). The reinfection threshold, marked by vertical dotted lines, is associated with 
a saturation of the trend observed for the susceptible $\left(\bar{\alpha}_{S}\right.$ appears constant for $R_{0}$ above threshold) and an average risk equal to one in the recovered class $\left(\bar{\alpha}_{R}=1\right)$. Compare with Figure 3. Overall, we have two equilibrium regimes. Below the reinfection threshold,

Figure 5: about here

the uninfected population is composed of many susceptible individuals with an average risk factor below one, and few recovered individuals with high risk due to selection imposed by infection. Above the reinfection threshold, most individuals have already experienced at least one infection and are still susceptible to reinfection but have an average risk factor below one. In the latter case, selection maintains a large proportion of the population in the infected class.

The patterns described for susceptible and recovered risk profiles have strong implications for the interpretation of disease dynamics, notably the contribution of reinfection to the overall disease incidence. We define the incidence of first infection and the incidence of reinfection, in the respective populations at risk, as

$$
\begin{gathered}
Y_{1}=\frac{\sigma \lambda\left(\alpha_{1} S_{1}^{*}+\alpha_{2} S_{2}^{*}\right)}{S^{*}}=\lambda \bar{\alpha}_{S} \\
Y_{2}=\frac{\sigma \lambda\left(\alpha_{1} R_{1}^{*}+\alpha_{2} R_{2}^{*}\right)}{R^{*}}=\sigma \lambda \bar{\alpha}_{R}
\end{gathered}
$$

The total incidence in the entire uninfected population is then calculated as

$$
Y_{\text {total }}=\left(Y_{1} S^{*}+Y_{2} R^{*}\right) /\left(S^{*}+R^{*}\right)
$$

Figure 6: about here

Figure 6 shows that despite reinfection being hindered by heterogeneity, the rate of reinfection among recovered individuals, $Y_{2}$, can be higher than overall rate of infection in the 
entire uninfected population, $Y_{\text {total }}$. We see that, for the homogeneous case $\left(\alpha_{1}=1\right)$, the quotient, $Y_{2} / Y_{\text {total }}$, increases monotonically with $R_{0}$, and for $R_{0}>1 / \sigma$ it is above one. For the heterogeneous case, reinfection among the recovered class can be higher than disease incidence also below the reinfection threshold. Even for low endemic populations, where the contribution of reinfection is low, it is possible that recovered individuals, as a group, show a higher risk of reinfection than expected when assuming partial immunity. This can have major implications for the interpretation of epidemiological data. In particular, overlooking host heterogeneity may lead to misleading expectations for the effectiveness of control measures.

\subsection{Contribution of the high-risk group}

As we have observed, for intermediate transmission levels, that reinfection occurs mainly in the high-risk group. So, it is also expected that its contribution to the overall transmission should be higher than in the heterogeneous SIR and SIS models.

Figure 7 shows the contribution of the high-risk group to the total disease prevalence for the particular case $\gamma=0.8$ and $\alpha_{1}=0.2$. This corresponds to a risk group of $20 \%$ of the total population with an increased risk of infection $\alpha_{2}=4.2$ times that of the total population, and 21 times that of the low-risk group. Moreover, for this choice of parameters, disease prevalence corresponds to about $30 \%$ of the homogeneous model prediction as represented by the dashed line in Figure 4(b) for $\sigma=0.25$. Here a sub-population of $20 \%$ accounts from $70 \%$ to $85 \%$ of the infection, depending on the intensity of transmission. The contribution of the high-risk group is stronger near the endemic and reinfection thresholds. Near the thresholds the classes that are susceptible to infection and reinfection, $S$ and $R$, respectively, reach their maximum capacity, accounting for almost all population. Therefore, the average risk on these classes and the selection pressure on the high-risk group are maximum.

Figure 7: about here

When considering heterogeneous infectivity, theoretical work and different field studies have suggested that roughly $20 \%$ of the infectious individuals can be responsible for $80 \%$ of 
transmission (Galvani \& May, 2005; Woolhouse et al., 1997). This 20/80 rule has important consequences for disease control (Woolhouse et al., 1997). Here we obtain similar effects by assuming heterogeneity in susceptibility to infection as previously estimated for the case of malaria transmission, where $20 \%$ of people receive $80 \%$ of all infections (Smith at al., 2005) due to heterogeneity in biting or in susceptibility to infection.

\section{Targeted vaccination}

The greater impact of the high-risk group on transmission should be taken into account when planning interventions for disease control. In this section, we compare uniform and targeted vaccination strategies. Comparison is made on the basis of the vaccination coverage required, under different strategies, to obtain the same impact. We implement vaccination at birth assuming that the protection conferred by the vaccine is equivalent to that of natural immunity. Vaccination reduces the risk of infection but the relative susceptibility of the two frailty groups is maintained. This is formalised as

$$
\left\{\begin{array}{l}
S_{i}^{\prime}=\left(1-v_{i}\right) \mu \gamma_{i}-\lambda \alpha_{i} S_{i}-\mu S_{i} \\
I_{i}^{\prime}=\lambda \alpha_{i} S_{i}+\sigma \lambda \alpha_{i} R_{i}-(\tau+\mu) I_{i} \\
R_{i}^{\prime}=v_{i} \mu \gamma_{i}+\tau I_{i}-\sigma \lambda \alpha_{i} R_{i}-\mu R_{i}, \quad i=1,2
\end{array}\right.
$$

The epidemic threshold for system (5.1) is described by

$$
R_{0}=\frac{1}{\left(\left(1-v_{1}\right)+\sigma v_{1}\right) \gamma \alpha_{1}+\left(\left(1-v_{2}\right)+\sigma v_{2}\right)(1-\gamma) \alpha_{2}}
$$

First, we consider a strategy based on a limited quantity of vaccines corresponding to a given coverage, $v$. We can then vary the percentage of each risk group covered by the program by fixing $v=v_{1} \gamma+v_{2}(1-\gamma)$ and varying $v_{2}$. Naturally, increasing the representation of the highrisk group in the vaccinated sub-population will increase the impact of the program (Britton, 1998). Here we reverse the argument and inspect what coverage we need to attain with a targeted strategy in order to achieve the same effectiveness as the corresponding uniform 
strategy. This will provide an estimation for how many doses we save by targeting the vaccination program to those individuals at higher risk, as a so called top-to-bottom strategy (Britton, 1998).

Figure 8: about here

Figure 8 illustrates the saving associated with targeting. For Figure $8(\mathrm{a})$ we use as a reference the reduction in disease prevalence achieved with a uniform vaccination strategy with coverage $v=0.5$. The figure shows the coverage for a targeted strategy to achieve the same reduction in disease prevalence. Below the reinfection threshold $\left(R_{0}=4\right)$ it is always possible to achieve the same reduction using a targeted strategy with lower coverage, while above the reinfection threshold there is no difference. Note that this is achieved by vaccinating only a proportion of the high-risk group (if $R_{0}$ is low enough - dotted line in the figure) or by vaccinating completely the high-risk group and a proportion of the low-risk group (dashed lines in the figure).

Figure 8(b) represents elimination coverages under different strategies. The critical vaccination coverage to eliminate the infection for a given $R_{0}$ is $v_{c}=\left(1-1 / R_{0}\right) /(1-\sigma)$ for the uniform strategy (full line) and $v_{c}=\left(1-1 / R_{0}\right)(1-\gamma) /\left[(1-\sigma)\left(1-\gamma \alpha_{1}\right)\right]$ or $v_{c}=$ $\left[\left(1-1 / R_{0}\right) /(1-\sigma)-\left(1-\alpha_{1}\right)\right] / \alpha_{1}$ for the top-to-bottom strategy with vaccination of only the high-risk group (dotted line) or both groups (dashed line), respectively. Under the reinfection threshold, the elimination coverage is always lower for the targeted strategy. Note that it is impossible to interrupt transmission and eliminate the infection above the reinfection threshold as previously described (Gomes et al., 2004, 2005). Above this threshold, only a superior vaccine, capable of inducing an immune response more effective than natural infection, would be efficacious.

\section{Discussion}

We have previously identified a reinfection threshold in the SIRI model and characterised how this induces a sharp division of the transmissibility axis into two regimes: reinfection is rare 
below threshold (SIR behavior) and very frequent above (SIS behavior). Here we describe how heterogeneity in innate susceptibility to infection smoothens this transition by making both regimes less extreme. Heterogeneity is always present in nature and it is important to understand how it can affect system behavior both qualitatively and quantitatively.

We perform a systematic analysis of the SIRI model with distributed susceptibility. The most striking result is the prediction that the average rate of reinfection may be higher than the average rate of primary infection, which may seem paradoxical given that primary infection induces life-long partial protection. The rationale behind this result is that infection generates a selection mechanism that skews the susceptibility profiles of the $S$ and $R$ compartments to lower and higher susceptibility, respectively. In other words, selection acts to keep fit individuals in $S$ and frail individuals in $R$. If this effect is strong enough we have a scenario where, on average, the rate of reinfection (infection out of $R$ ) is higher than the rate of primary infection (infection out of $S$ ) even though each individual has a risk reduction following primary infection. This mechanism may explain high rates of tuberculosis reinfection recently reported (Verver et al., 2005).

A rule of thumb has been proposed in infections disease dynamics, whereby $20 \%$ of the population is responsible for $80 \%$ of all infections due to heterogeneity in susceptibility or infectivity (Woolhouse et al., 1997). However, direct confirmation of this hypothesis requires very large epidemiological studies. For diseases that induce partial immunity, mathematical models such as those proposed here offer the practical alternative of using the ratio between reinfection and primary infection rates as an indirect measure of population heterogeneity.

In the SIRI models with heterogeneous susceptibility, we predict that disease prevalence is lower than the corresponding homogeneous model, as described before for epidemic SIR models (Gart, 1968; Ball, 1985; Anderson \& Britton, 1998; Miller, 2007). In other words, to obtain a given level of disease prevalence, the heterogeneous model requires a higher value for the transmission intensity, $R_{0}$. This implies that elimination strategies require more effort under wider heterogeneity (Anderson \& May, 1991).

The success of vaccination depends then on the ability to target those individuals at higher groups. Generally, there is an additional benefit associated with targeting vaccination 
strategies, as previously described for the SIR epidemic model (Britton, 1998; Koopman et al., 2005). In the case of the SIRI model, however, the added value of targeting high-risk groups is limited to those regions where transmission is below the reinfection threshold.

\section{Acknowledgments}

Research funded by the Gulbenkian Foundation (FCG), the Portuguese Research Council (FCT), ISFL/1/209 and the FCT Grant SFRH/BD/30168/2006 and the European Commission Grant MEXT-CT-2004-14338.

\section{Appendix}

\section{A Proofs of Section 3.1}

For $\sigma=0$ (SIR model), from system (2.1) we obtain

$$
\left\{\begin{array}{l}
S_{1}^{\prime}=\mu \gamma-\beta I \alpha_{1} S_{1}-\mu S_{1} \\
S_{2}^{\prime}=\mu(1-\gamma)-\beta I \alpha_{2} S_{2}-\mu S_{2} \\
I^{\prime}=\beta I\left(\alpha_{1} S_{1}+\alpha_{2} S_{2}\right)-(\tau+\mu) I
\end{array}\right.
$$

When $\sigma=1$ (SIS model), we can collapse the recovered classes into the susceptible ones in system (2.1). Then, if we denote for simplicity by $S_{i}$ the classes $S_{i}+R_{i}, i=1,2$, and use the fact that $\gamma_{i}=S_{i}+I_{i}$, we obtain the following system

$$
\left\{\begin{aligned}
S_{1}^{\prime} & =(\tau+\mu) \gamma-\beta I \alpha_{1} S_{1}-(\tau+\mu) S_{1} \\
S_{2}^{\prime} & =(\tau+\mu)(1-\gamma)-\beta I \alpha_{2} S_{2}-(\tau+\mu) S_{2} \\
I^{\prime} & =\beta I\left(\alpha_{1} S_{1}+\alpha_{2} S_{2}\right)-(\tau+\mu) I .
\end{aligned}\right.
$$

Note that system (A.2) is equivalent to an SIR model where the birth and death rate are equal to $\tilde{\mu}=\tau+\mu$ and the recovery rate is $\tilde{\tau}=0$. 


\section{Proof of Theorem 3.1.1}

Proof. Let us first consider $\sigma=0$. The Jacobian of system (A.1) at the disease-free equilibrium is

$$
J\left(E_{0}\right)=\left[\begin{array}{ccc}
-\mu & 0 & -\beta \alpha_{1} \gamma \\
0 & -\mu & -\beta \alpha_{2}(1-\gamma) \\
0 & 0 & \beta-(\tau+\mu)
\end{array}\right]
$$

The eigenvalues of this matrix are $-\mu$ and $\beta-(\tau+\mu)$. So we conclude that $E_{0}$ is locally asymptotically stable for $R_{0}<1$ and unstable for $R_{0}>1$. Moreover, system (A.1) is equivalent to system (3.1) in Hyman \& Li (2005) for $n=2$. In Theorem 3.1 of that paper, the authors prove the global stability for the disease-free equilibrium for $R_{0}<1$.

For the case $\sigma=1$, calculations can be repeated using $\tilde{\mu}=\tau+\mu$ as the new birth and death rates and $\tilde{\tau}=0$ as the new rate of recovery.

\section{Proof of Theorem 3.1.2}

Proof. The second member of system (A.1) vanishes at the equilibria. From the two first equations we get a relation between $S_{i}$ and $I: S_{1}=\gamma \mu /\left(\mu+\beta I \alpha_{1}\right)$ and $S_{2}=(1-\gamma) \mu /(\mu+$ $\left.\beta I \alpha_{2}\right)$. Substituting in the third one we get $(\tau+\mu) \frac{P(I)}{Q(I)} I=0$, where $P(I)=a_{2} I^{2}+a_{1} I+a_{0}$ with $a_{2}=-\alpha_{1} \alpha_{2} R_{0}^{2}(\tau+\mu)^{2}, a_{1}=R_{0}(\tau+\mu) \mu\left(\alpha_{1} \alpha_{2} R_{0}-\left(\alpha_{1}+\alpha_{2}\right)\right)$ and $a_{0}=\mu^{2}\left(R_{0}-1\right)$ and $Q(I)=\left(\mu+\beta I \alpha_{1}\right)\left(\mu+\beta I \alpha_{2}\right)$. Note that for $I \geq 0$ we have $Q(I)>0$. We conclude that the $I$ coordinate of the nontrivial equilibria of system (A.1) will correspond to a positive solution of $P(I)=0$. Since $a_{2}<0$ and $a_{0}>0$ for $R_{0}>1$ we conclude that the polynomial $P$ has exactly one positive solution of the form:

$$
I^{0}\left(R_{0}\right)=\frac{-a_{1}-\sqrt{a_{1}^{2}-4 a_{2} a_{0}}}{2 a_{2}}
$$

and this proves the first part of the theorem.

In what concerns stability, system (A.1) is equivalent to system (3.1) in Hyman \& Li (2005) for $n=2$. In Theorem 3.2 of that paper, the authors prove the stability for the endemic equilibrium for $R_{0}>1$ via Liapunov stability theory. 
As in the previous proof, for the case $\sigma=1$, calculations can be repeated using $\tilde{\mu}=\tau+\mu$ as the new birth and death rates and $\tilde{\tau}=0$ as the new rate of recovery.

Remark A.1. From the proof of theorem 3.1.2 it is possible to establish a relation between the disease prevalence of both models. In fact, for every $\gamma \in(0,1)$ and $\alpha_{1} \in(0,1]$ we have

$$
I^{1}=\frac{\tau+\mu}{\mu} I^{0}
$$

where $I^{0}$ and $I^{1}$ represent the disease prevalence at equilibrium for the SIR and SIS models, respectively. This relation is systematically used to extend the proofs from the case $\sigma=0$ to the case $\sigma=1$.

We can also conclude that for all $\gamma \in(0,1)$ and $\alpha_{1} \in(0,1]$

$$
\lim _{R_{0} \rightarrow+\infty} I^{0}=\frac{\mu}{\tau+\mu} \text { and } \lim _{R_{0} \rightarrow+\infty} I^{1}=1 .
$$

Remark A.2. From the proof of Theorem 3.1.2, taking into account Remark A.1, we recover the expression of the endemic equilibrium for the homogeneous model, both for the $\sigma=0$ and the $\sigma=1$ cases, by using $\alpha_{1}=\alpha_{2}=1$ (or $\alpha_{1}=1$ ):

$$
I_{\text {Hom }}^{0}\left(R_{0}\right)=\frac{\mu}{\tau+\mu}\left(1-\frac{1}{R_{0}}\right) \text { and } I_{\text {Hom }}^{1}\left(R_{0}\right)=1-\frac{1}{R_{0}} .
$$

\section{Proof of Theorem 3.1.3}

Proof. First, let $\sigma=0$ and denote by $I^{0}$ and $I^{0^{*}}$, respectively, the unique positive and negative roots of the polynomial $P$ defined in the proof of Theorem 3.1.2. Differentiating $P\left(I^{0}\right)=0$ with respect to a parameter $\epsilon$, we get

$$
\frac{\partial I^{0}}{\partial \epsilon}=\frac{-\frac{\partial a_{2}}{\partial \epsilon} I^{0^{2}}-\frac{\partial a_{1}}{\partial \epsilon} I^{0}-\frac{\partial a_{0}}{\partial \epsilon}}{2 a_{2} I^{0}+a_{1}}
$$

Note that we have $I^{0}+I^{0 *}=-a_{1} / a_{2}$ and that $a_{2}<0$. Hence we conclude that the denominator of $\left(\right.$ A.7) verifies $2 a_{2} I^{0}+a_{1}=a_{2}\left(2 I^{0}+a_{1} / a_{2}\right)=a_{2}\left(2 I^{0}-I^{0}-I^{0 *}\right)=a_{2}\left(I^{0}-I^{0 *}\right)<0$. As 
a consequence of this fact, the sign of (A.7) will be the opposite of the one of the numerator.

Let $\epsilon=\gamma$. In this case, $\partial a_{0} / \partial \gamma=0$. So, $\partial I^{0} / \partial \gamma \leq 0$ iff $\frac{\partial a_{2}}{\partial \gamma} I^{0}+\frac{\partial a_{1}}{\partial \gamma}<0$. Now we replace $I^{0}$ by its expression in (A.3). Since $a_{2}<0$ and $\partial a_{2} / \partial \gamma<0$ we get the following equivalent condition $-a_{1}+2 a_{2} \frac{\partial a_{1}}{\partial \gamma} / \frac{\partial a_{2}}{\partial \gamma}<\sqrt{a_{1}^{2}-4 a_{0} a_{2}}$. If the left hand side is negative, then the condition is true and the result is proved. Otherwise, we can square both sides. In this case we get $\left(-a_{1}+2 a_{2} \frac{\partial a_{1}}{\partial \gamma} / \frac{\partial a_{2}}{\partial \gamma}\right)^{2}-a_{1}^{2}+4 a_{0} a_{2}=-4 \alpha_{1} R_{0}(1-\gamma)(1-\alpha)\left(1-\gamma \alpha_{1}\right)<0$, which ends this part of the proof.

Let $\epsilon=\alpha_{1}$. Now we have $\partial I^{0} / \partial \alpha_{1} \geq 0$ iff

$$
\frac{\partial a_{2}}{\partial \alpha_{1}} I^{0}+\frac{\partial a_{1}}{\partial \alpha_{1}}>0
$$

Again, we substitute $I^{0}$ by its expression in (A.3). Depending on the sign of $\partial a_{2} / \partial \alpha_{1}(\neq 0)$ we obtain two different cases:

Case 1. If $\frac{\partial a_{2}}{\partial \alpha_{1}}<0$ then (A.8) is equivalent to $-a_{1}+2 a_{2} \frac{\partial a_{1}}{\partial \alpha_{1}} / \frac{\partial a_{2}}{\partial \alpha_{1}}>\sqrt{a_{1}^{2}-4 a_{0} a_{2}} \quad(a)$

Case 2. If $\frac{\partial a_{2}}{\partial \alpha_{1}}>0$ then (A.8) is equivalent to $-a_{1}+2 a_{2} \frac{\partial a_{1}}{\partial \alpha_{1}} / \frac{\partial a_{2}}{\partial \alpha_{1}}<\sqrt{a_{1}^{2}-4 a_{0} a_{2}}$ (b).

Note that if $\partial a_{2} / \partial \alpha_{1}=0$ then we must see if $\partial a_{1} / \partial \alpha_{1}>0$, which is true for $R_{0}>1$.

The sign of $\partial a_{2} / \partial \alpha_{1}$ is the same as the sign of $2 \alpha_{1} \gamma-1$. So, for case 1 , let us assume $2 \alpha_{1} \gamma<1$. The left hand side of (a) is $\frac{R_{0}(\tau+\mu) \mu\left[R_{0} \alpha_{1}\left(\left(1-\alpha_{1} \gamma\right)\left(1-2 \alpha_{1} \gamma\right)\right)+1-\alpha_{1}\right]}{(1-\gamma)\left(1-2 \alpha_{1} \gamma\right)}$ which is positive for $2 \alpha_{1} \gamma>1$. Hence, we can square both sides of (a) and we get $\left(-a_{1}+\right.$ $\left.2 a_{2} \frac{\partial a_{1}}{\partial \gamma} / \frac{\partial a_{2}}{\partial \gamma}\right)^{2}-a_{1}^{2}+4 a_{0} a_{2}=\frac{R_{0}^{2}(\tau+\mu)^{2} \mu^{2} 4 \gamma \alpha_{1}}{(1-\gamma)^{2}\left(1-2 \alpha_{1} \gamma\right)^{2}}\left(1-\alpha_{1}\right)\left(1-\alpha_{1} \gamma\right)\left[R_{0}\left(\left(1-2 \alpha_{1} \gamma\right)^{2}+\alpha_{1}(1-\right.\right.$ $\left.\left.\left.2 \alpha_{1} \gamma\right)\right)+1-\alpha_{1}\right]>0$ which ends the proof of case 1 .

In case 2 the left hand side can change sign. Let us denote the left hand side by $B$. So, to verify (b) we have to show that if $B$ is positive then $B^{2}-a_{1}^{2}+4 a_{0} a_{2}<0$. By the calculations for the previous case we get that, for $2 \alpha_{1} \gamma>1, B>0$ iff $C=R_{0} \alpha_{1}\left(\left(1-\alpha_{1} \gamma\right)\left(1-2 \alpha_{1} \gamma\right)\right)+1-\alpha_{1}<$ 0 . Again, from the previous case we know that the sign of $B^{2}-a_{1}^{2}+4 a_{0} a_{2}$ is the same as the one of $R_{0}\left(\left(1-2 \alpha_{1} \gamma\right)^{2}+\alpha_{1}\left(1-2 \alpha_{1} \gamma\right)\right)+1-\alpha_{1}=C / \alpha_{1}+R_{0}\left(1-2 \alpha_{1} \gamma\right) \alpha_{1}(1-\gamma)-\left(1-\alpha_{1}\right)^{2} / \alpha_{1}<0$, since $C<0$. This ends the proof of case (b).

Finally, let $\epsilon=R_{0}$. In this case $\partial a_{0} / \partial R_{0} \neq 0$. So, from (A.7) and since the denominator 
is non-positive, to prove that $\partial I^{0} / \partial R_{0} \geq 0$ we need to prove that

$$
\frac{\partial a_{2}}{\partial R_{0}} I^{0^{2}}+\frac{\partial a_{1}}{\partial R_{0}} I^{0}+\frac{\partial a_{0}}{\partial R_{0}}>0
$$

Now, taking into account that $I^{0^{2}}=-\left(a_{1} I^{0}+a_{0}\right) / a_{2}$ and using the expression of $I_{0}$ given in (A.3) we obtain $-A a_{1}+2 a_{2} B>A \sqrt{a_{1}^{2}-4 a_{0} a_{2}}$, where $A=\frac{\partial a_{1}}{\partial R_{0}} a_{2}-\frac{\partial a_{2}}{\partial R_{0}} a_{1}$ and $B=$ $a_{2} \frac{\partial a_{0}}{\partial R_{0}}-a_{0} \frac{\partial a_{2}}{\partial R_{0}}$. By substituting $a_{i}$ and its derivatives in $A$ we conclude that $A=-\alpha_{1}(1-$ $\left.\alpha_{1} \gamma\right) R_{0}^{2}(\tau+\mu)^{3} \mu\left(\alpha_{1}(1-\gamma)+\left(1-\alpha_{1} \gamma\right)\right) /(1-\gamma)^{2}<0$. So, we can divide both sides by $\mathrm{A}$ and get

$$
-a_{1}+2 a_{2} B / A>\sqrt{a_{1}^{2}-4 a_{0} a_{2}}
$$

If the left hand side is negative, then the condition is true and the result is proved. Otherwise, we can square both sides of (A.10). Finally, we need to prove that the following expression $-4 C\left(1-\alpha_{1} \gamma\right) \alpha_{1} \mu^{2}(\tau+\mu)^{2} R_{0}^{2} /(1-\gamma)\left(\alpha_{1}(1-\gamma)+\left(1-\alpha_{1} \gamma\right)\right)^{2}$ is negative, where $C$ is a polynomial in $R_{0}$ of degree 2 with coefficients $c_{2}=\alpha_{1}\left(1-\alpha_{1} \gamma\right)\left(\alpha_{1}(1-\gamma)+\gamma\left(1-\alpha_{1}\right)\right)$, $c_{1}=2 \alpha_{1}\left(1-\alpha_{1}\right)\left(1-\alpha_{1} \gamma\right)(1-2 \gamma)$ and $c_{0}=\left(1-\alpha_{1}\right)^{2}$. We note that, the minimum value of $C$ is attained at $R_{0}^{m}=1-(1-\gamma) /\left(\alpha_{1}(1-\gamma)+\gamma\left(1-\alpha_{1}\right)\right)<1$ and $C$ is positive at $R_{0}^{m}$. As a consequence, since $c_{2}>0$ we conclude that $C$ is positive for $R_{0}>1$. This concludes this part of the proof.

For $\sigma=1$ the proofs follow easily from Remark A.1.

\section{B Proofs of Section 3.2}

First we derive an expression which relates the disease prevalence with the relative risk of the susceptible class in the case of the SIR model and the average risk of infection of the $S+R$ class in the case of the SIS system. Then we can use the results from the previous section to prove theorem 3.2.1 and to get Remark 3.1. 
In the SIR case, from (A.1) letting $S=S_{1}+S_{2}$, we obtain

$$
\left\{\begin{array}{l}
S^{\prime}=\mu-\beta I \bar{\alpha}_{S}^{0} S-\mu S \\
I^{\prime}=\beta I \bar{\alpha}_{S}^{0} S-(\tau+\mu) I
\end{array}\right.
$$

Hence, we get an implicit expression for the disease prevalence at equilibrium in the case $\sigma=0$

$$
I^{0}=\frac{\mu}{\tau+\mu}\left(1-\frac{1}{\bar{\alpha}_{S}^{0} R_{0}}\right)
$$

Similarly, from system (A.2), we obtain the relation between the disease prevalence $I^{1}$ and the average risk of infection

$$
I^{1}=1-\frac{1}{\bar{\alpha}_{S+R}^{1} R_{0}} .
$$

Remark B.1. In particular, from Remark A.1 we conclude by this last equality that $\bar{\alpha}_{S}^{0}=$ $\bar{\alpha}_{S+R}^{1}$.

\section{Proof of Theorem 3.2.1}

Proof. Let $\sigma=0$. From (B.2) we obtain the following expression for $\bar{\alpha}_{S}^{0}$

$$
\bar{\alpha}_{S}^{0}=\frac{\mu}{R_{0}\left(\mu-(\tau+\mu) I^{0}\right)}
$$

Thus, for $\epsilon=\gamma$ or $\alpha_{1}$ we get

$$
\frac{\partial \bar{\alpha}_{S}^{0}}{\partial \epsilon}=\frac{\mu(\tau+\mu)}{R_{0}\left[\mu-(\tau+\mu) I^{0}\right]^{2}} \frac{\partial I^{0}}{\partial \epsilon}
$$

which has the same sign as $\partial I^{0} / \partial \epsilon$.

For the derivative of $\bar{\alpha}_{S}^{0}$ with respect to $R_{0}$ we get

$$
\frac{\partial \bar{\alpha}_{S}^{0}}{\partial R_{0}}=\frac{\mu}{\left[R_{0}\left(\mu-(\tau+\mu) I^{0}\right)\right]^{2}}\left[\mu-(\tau+\mu) I^{0}-R_{0}(\tau+\mu) \frac{\partial I^{0}}{\partial R_{0}}\right]
$$

Hence, to prove that the derivative is negative is equivalent to prove that $I^{0}+\partial I^{0} / \partial R_{0}<$ $\mu /(\tau+\mu)$. Now we substitute $\left(I^{0}\right)^{2}$ by $-\left(a_{1} I^{0}+a_{0}\right) / a_{2}$ and $\partial I^{0} / \partial R_{0}$ by the expression from 
(A.7). Furthermore, we replace $I^{0}$ by its expression in (A.3). So, taking into account that $a_{2}<$ 0 and $A=R_{0} \frac{\partial a_{2}}{\partial R_{0}} a_{1}-a_{2} a_{1}-\frac{\partial a_{1}}{\partial R_{0}} a_{2} R_{0}-2 a_{2}^{2} \mu /(\tau+\mu)=-\mu(\tau+\mu)^{3} \alpha_{1}^{2}\left(1-\alpha_{1} \gamma\right)^{2} R_{0}^{4} /(1-\gamma)^{2}<0$ we get to the equivalent condition

$$
-a_{1}+2 a_{2} B / A<\sqrt{a_{1}^{2}-4 a_{0} a_{2}}
$$

If the left hand side is negative then the condition is true. Otherwise we can square both sides of (B.7). Hence $\left(-a_{1}+2 a_{2} B / A\right)^{2}-a_{1}^{2}+4 a_{0} a_{2}=-4 R_{0}^{2} \gamma \mu^{2}(\tau+\mu)^{2}\left(1-\alpha_{1}\right)^{2} /(1-\gamma)<0$. which ends this part of the proof.

\section{Proof of Theorem 3.2.2}

Proof. As for the previous proofs, we start by studying the case $\sigma=0$ and then the case $\sigma=1$ follows directly from Remark A.1. In fact, in this case we have $Q^{0}=Q^{1}$.

For simplicity we write $Q^{0}=I_{2}^{0} / I^{0}$ as $1-I_{1}^{0} / I^{0}=1-R_{0} \alpha_{1} \mu \gamma /\left(R_{0}(\tau+\mu) I^{0} \alpha_{1}+\mu\right)$. The derivative of $Q^{0}$ with respect to $\gamma$ is

$$
\frac{\partial Q^{0}}{\partial \gamma}=-\frac{R_{0} \alpha_{1} \mu\left[\mu+R_{0} \alpha_{1}(\tau+\mu)\left(I^{0}-\gamma \frac{\partial I^{0}}{\partial \gamma}\right)\right]}{\left(R_{0}(\tau+\mu) I^{0} \alpha_{1}+\mu\right)^{2}} .
$$

But $I^{0}-\gamma \frac{\partial I^{0}}{\partial \gamma}=I^{0}\left(1+\gamma \frac{\frac{\partial a_{2}}{\partial \gamma} I^{0}+\frac{\partial a_{1}}{\partial \gamma}}{2 a_{2} I^{0}+a_{1}}\right) \geq 0$ from what was seen in the proof of Theorem 3.1.3. Thus we conclude that $\frac{\partial Q^{0}}{\partial \gamma} \leq 0$.

The derivative of $Q^{0}$ with respect to $\alpha_{1}$ is

$$
\frac{\partial Q^{0}}{\partial \alpha_{1}}=-\frac{R_{0} \gamma \mu\left[\mu-R_{0} \alpha_{1}^{2}(\tau+\mu) \frac{\partial I^{0}}{\partial \alpha_{1}}\right]}{\left(R_{0}(\tau+\mu) I^{0} \alpha_{1}+\mu\right)^{2}} .
$$

This expression has the opposite sign of $C=\mu-R_{0} \alpha_{1}^{2}(\tau+\mu) \frac{\partial I^{0}}{\partial \alpha_{1}}$. Again, we replace $I^{0^{2}}$ by $-\left(a_{1} I^{0}+a_{0}\right) / a_{2}$ and then $I^{0}$ by its expression in (A.3). Finally, we conclude that $C \geq 0$ iff $-a_{1} A+2 a_{2} B \leq A \sqrt{a_{1}^{2}+4 a_{2} a_{0}}$, where $A=2 \mu a_{2}^{2}-R_{0} \alpha_{1}^{2}(\tau+\mu) \frac{\partial a_{2}}{\partial \alpha_{1}} a_{1}+R_{0} \alpha_{1}^{2}(\tau+\mu) \frac{\partial a_{1}}{\partial \alpha_{1}} a_{2}$ and $B=a_{1} a_{2} \mu-R_{0} \alpha_{1}^{2}(\tau+\mu) \frac{\partial a_{2}}{\partial \alpha_{1}} a_{0}$. Note that by substituting $a_{i}$ and its derivatives in $A$ we 
can easily conclude that $A$ is positive. Therefore, we can divide both sides by $A$, obtaining that $C \geq 0$ iff

$$
-a_{1}+2 a_{2} B / A \leq \sqrt{a_{1}^{2}+4 a_{2} a_{0}}
$$

If the left hand side is negative, then the condition is verified. Otherwise we can square both sides of (B.10). Hence, we get $-\frac{4\left(1-\alpha_{1} \gamma\right)^{2} \gamma \alpha_{1}^{2} \mu^{2}(\tau+\mu)^{2} R_{0}^{4}}{(1-\gamma)} \leq 0$. This implies that $C \geq 0$ or, equivalently, that $\partial Q^{0} / \partial \alpha_{1} \leq 0$, which ends this part of the proof.

The derivative of $Q^{0}$ with respect to $R_{0}$ is

$$
\frac{\partial Q^{0}}{\partial R_{0}}=\frac{\alpha_{1} \gamma \mu\left[\mu-R_{0}^{2} \alpha_{1}(\tau+\mu) \frac{\partial I^{0}}{\partial R_{0}}\right]}{\left(R_{0}(\tau+\mu) I^{0} \alpha_{1}+\mu\right)^{2}}
$$

This expression has the same sign of $C=b-R_{0}^{2} \frac{\partial I^{0}}{\partial R_{0}}$, where $b=\mu /\left(\alpha_{1}(\tau+\mu)\right)$. We conclude that $C>0$ iff $-a_{1} A+2 a_{2} B>A \sqrt{a_{1}^{2}+4 a_{2} a_{0}}$, where $A=\frac{\partial a_{2}}{\partial R_{0}} a_{1}-\frac{\partial a_{1}}{\partial R_{0}} a_{2}-2 a_{2}^{2} b^{\prime}, B=$ $a_{0} \frac{\partial a_{2}}{\partial R_{0}}-\frac{\partial a_{0}}{\partial R_{0}} a_{2}-b^{\prime} a_{1} a_{2}$ and $b^{\prime}=b a_{2} / R_{0}^{2}$. Note that by substituting $a_{i}$ and its derivatives in $A$ we can easily conclude that $A$ is negative. So, we can divide both sides by $A$, obtaining that $C>0$ iff

$$
-a_{1}+2 a_{2} B / A<\sqrt{a_{1}^{2}+4 a_{2} a_{0}}
$$

If the left hand side is negative the condition is verified. Otherwise we can square both sides of (B.12). Hence, we get $-\frac{4\left(1-\alpha_{1} \gamma\right)^{2} \gamma \alpha_{1}^{2} \mu^{2}(\tau+\mu)^{2} R_{0}^{4}}{(1-\gamma)}<0$. This ends the proof.

\section{The reinfection threshold for the heterogeneous SIRI model}

To compute the reinfection threshold associated with the heterogeneous SIRI model (2.1) with $n=2$, we first have to define the reinfection sub-model. This model corresponds to the limit situation where all individuals that enter in the system are partiality immunized and only subjected to reinfection. Hence, the reinfection sub-model has only four classes and can 
be represented with the following system of differential equations:

$$
\left\{\begin{aligned}
R_{1}^{\prime} & =\mu \gamma+\tau I_{1}-\sigma \lambda \alpha_{1} R_{1}-\mu R_{1} \\
I_{1}^{\prime} & =\sigma \lambda \alpha_{1} R_{1}-(\tau+\mu) I_{1} \\
R_{2}^{\prime} & =\mu(1-\gamma)+\tau I_{2}-\sigma \lambda \alpha_{2} R_{2}-\mu R_{2} \\
I_{2}^{\prime} & =\sigma \lambda \alpha_{2} R_{2}-(\tau+\mu) I_{2}
\end{aligned}\right.
$$

The reinfection sub-model has a unique disease-free equilibrium $E_{0}=(\gamma, 0,1-\gamma, 0)$. Analyzing the jacobian at $E_{0}$ we conclude that a bifurcation on the transmission parameter $\beta$ occurs at $\beta=(\tau+\mu) / \sigma$ when the disease free equilibrium changes its stability. In terms of the basic reproduction number the bifurcation is attained at $R_{0}=1 / \sigma$.

\section{References}

Anderson, H., Britton, T., 1998. Heterogeneity in epidemic models and its effect on the spread of infection. J. Appl. Prob. 35, 651-61.

Anderson R.M., May R.M., 1985. Age-related changes in the rate of disease transmission: implications for the design of vaccination programmes. J Hyg (Lond). 94(3), 365-436.

Anderson, R.M., May, R.M., 1991. Infectious diseases of humans. Oxford Science Publication, Oxford.

Ball, F., 1985. Deterministic and stochastic epidemics with several kinds of susceptibles. Adv. Appl. Prob. 17(1), 1-22.

Britton, T., 1998. On critical vaccination coverage in multiple epidemics. J. Appl. Prob. 35, 1003-6.

Coutinho, F.A.B., Massad, E., Lopez, L.F., Burattini, M.N., Struchiner, C.J., Azevedo- 
Neto, R.S., 1999. Modelling heterogeneities in individual frailties in epidemic models. Math. Comp. Mod. 30, 97-115

Diekmann, O., Heesterbeek, J.A.P., Metz, J.A.J., 1990. On the definition and computation of the basic reproduction ratio $R_{0}$ in models for infectious diseases in heterogeneous populations. J. Math. Biol. 28, 365-382.

Fine PE, Clarkson JA., 1982. Measles in England and Wales-II: The impact of the measles vaccination programme on the distribution of immunity in the population. Int J Epidemiol. $11(1), 15-25$.

Galvani, A., May, R.M., 2005. Dimensions of superspreading. Nature. 438, 293-5.

Gart, J.J., 1968. The mathematical analysis of an epidemic with two kinds of susceptibles. Biometrics. 24, 557-66.

Gomes, M.G.M., White, L.J., Medley, G.F., 2004. Infection, reinfection, and vaccination under suboptimal immune protection: epidemiological perspectives. J. Theor. Biol. 235(2), 151-2., doi:10.1016/j.jtbi.2004.02.015.

Gomes, M.G.M., White, L.J., Medley, G.F., 2005. The reinfection threshold. J. Theor. Biol. 236, 111-13., doi:10.1016/j.jtbi.2005.03.001.

Hyman, M.J., Li, J., 2000. An intuitive formulation for the reproductive number for the spread of diseases in heterogeneous populations. Math. Biosc. 167, 65-86., doi:10.1016/S0025$5564(00) 00025-0$.

Hyman, M.J., Li, J., 2005. Differential susceptibility epidemic models. J. Math. Biol. 50, 626-44.

Koopman, J.S., Simon, C.P., Riolo, C.P., 2005. When to control endemic infections by focusing on high-risk groups. Epidemiology. 16(5), 621-27.

Miller, J.C., 2007. Epidemic size and probability in populations with heterogeneous infectivity and susceptibility. Phys. Rev. E. 76, [Epub], doi:10.1103/PhysRevE.76.010101. 
Smith, D.L., Dushoff, J., Snow, R.W., Hay, S.I., 2005. The entomological inoculation rate and Plasmodium falciparum infection in Africa children. Nature. 438(24), 492-5.

van Boven, M., de Melker, H.E., Schellekens, J.F.P., Kretzschmar, M., 2001. A model based evaluation of the 1996-7 pertussis epidemic in the Netherlands. Epidemiol. Infect. 127, 73-85.

Verver, S., Warren, R., Beyers, N., Richardson, M., van der Spuy, G., Borgdorff, M.W., Enarson, D.A., Behr, M.A., van Helden P.D., 2005. Rate of reinfection tuberculosis after successful treatment is higher than rate of new tuberculosis. Am. J. Respir. Crit. Care Med. $171,1430-35$.

Woolhouse, M.E.J., Dye, C., Etard, J.F., Smith, T., Charlwood, J.D., Garnett, G.P., Hagan, P., Hii, J.L.K., Ndhlovu, P.D., Quinnell, R.J., Watts, C.H., Chandiwana, S.K., Anderson, R.M., 1997. Heterogeneities in the transmission of infectious agents: implications for the design control programs. Proc. Natl. Acad. Sci. USA 94, 338-42. 


\section{List of Figures}

1 SIRI model with heterogeneous susceptibility to infection. The population is divided into Susceptible $\left(S_{i}\right)$, Infectious $\left(I_{i}\right)$ and Recovered $\left(R_{i}\right)$ classes, where the index $i$ refers to the frailty group to which the individuals belong. Individuals are born at rate $\mu$ and enter the susceptible compartments in proportions $\gamma_{i}$. susceptible individuals are infected at a rate $\alpha_{i} \lambda=\alpha_{i} \beta I$, where $\alpha_{i}$ denotes the risk factor, $\beta$ is the transmission coefficient and $I$ is the proportion of infectious individuals. Infectious individuals recover at a rate $\tau$ and recovered individuals have a reduced rate of reinfection according to the factor $\sigma$. . . . . .

2 Prevalence of infection for the SIR and SIS models under different implementations of two risk groups (low and high). Top and bottom pannels correspond to the SIR and SIS models, respectively: (a),(b),(c) $\sigma=0 ;(\mathrm{d}),(\mathrm{e}),(\mathrm{f}) \sigma=1$. The three columns of pannels correspond to different proportions of population at low risk: (a),(d) $\gamma=0.5$; (b),(e) $\gamma=0.8 ;$ (c),(f) $\gamma=0.95$. In each plot, different curves indicate the equilibrium prevalence of infection under different susceptibility ratios between the low-risk group and the average: $\alpha_{1}=1,0.75,0.5,0.2,0.05$, from the higher to the lower curves. $\ldots \ldots \ldots$

3 Average risk factor before and after infection. (a),(b) Contour plots for $\bar{\alpha}_{S}^{0}, \bar{\alpha}_{R}^{0}$ in the SIR model; (c),(d) Contour plots for $\bar{\alpha}_{S}^{1}, \bar{\alpha}_{R}^{1}$ in the SIS model. Contours are represented in terms of the basic reproduction number, $R_{0}$, and the relative susceptibility of the low risk group, $\alpha_{1}$. The proportion at low risk is $\gamma=0.8$ in both cases. . . . . . . . .

4 Prevalence of infection for the SIRI model under different implementations of two risk groups (low and high). The three pannels correspond to different proportions of the population at risk: (a),(d) $\gamma=0.5$; (b),(e) $\gamma=0.8$; (c),(f) $\gamma=0.95$. In each plot, different curves indicate the equilibrium prevalence of infection under different susceptibility ratios between the low-risk group and the average: $\alpha_{1}=1,0.75,0.5,0.2,0.05$, from the higher to the lower curves. . . . . . . . . . . . . . . . 
$5 \quad$ Average risk factor for susceptible and recovered classes in the SIRI model. Contour plots for: (a) the susceptible class, $\bar{\alpha}_{S}^{\sigma}$; and (b) the recovered class, $\bar{\alpha}_{R}^{\sigma}$. Contours are represented in terms of the basic reproduction number, $R_{0}$, and the relative susceptibility of the low risk group, $\alpha_{1}$. The proportion at low risk is $\gamma=0.8$ and susceptibility reduction due to partial immunity is $\sigma=0.25 \ldots \ldots \ldots \ldots \ldots$

6 Intensity of reinfection. (a) Rate of reinfection among recovered individuals over the total incidence. (b) Rate of reinfection among recovered individuals over the incidence of first infection. Different values are considered for the relative risk of the low risk group, $\alpha_{1}$, including the homogeneous case, $\alpha_{1}=1$. The proportion of the population at low risk, $\gamma$, is

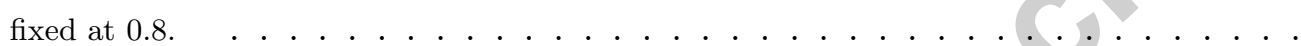

7 Contribution of the high-risk group to the total disease prevalence. Infection risk distribution is given by $\gamma=0.8$ and $\alpha_{1}=0.2$. The dashed line corresponds to both $\sigma=0$ and $\sigma=1$, while other lines correspond to values of $\sigma$ as indicated. The special case $\sigma=0.25$ is distinguished as a full line as this parameter values is used for illustration in other figures. The horizontal line represents the case for which the infection risk distribution is homogeneous $\left(\alpha_{1}=\alpha_{2}=1\right)$. . . . . . . . . . . . . . . . . . . . . . .

8 Uniform vs. targeted vaccination programs. (a) Vaccination coverage required for the targeted strategy to obtain the same disease reduction as a uniform strategy with $50 \%$ coverage, for each $R_{0}$. (b) Vaccination coverage required to eliminate the infection for each $R_{0}$. Full lines corresponds to the uniform strategy and broken lines correspond to the targeted strategy (dotted if vaccination is restricted to the high-risk group, and dashed if this is complemented by vaccination in the low-risk group). Infection risk distribution is given by $\gamma=0.8$ and $\alpha_{1}=0.2$. . . . . . . . . . . . . . . . . . . . . . . . . . . .

\section{List of Tables}

$1 \quad$ Model parameters . . . . . . . . . . . . . . . . . 

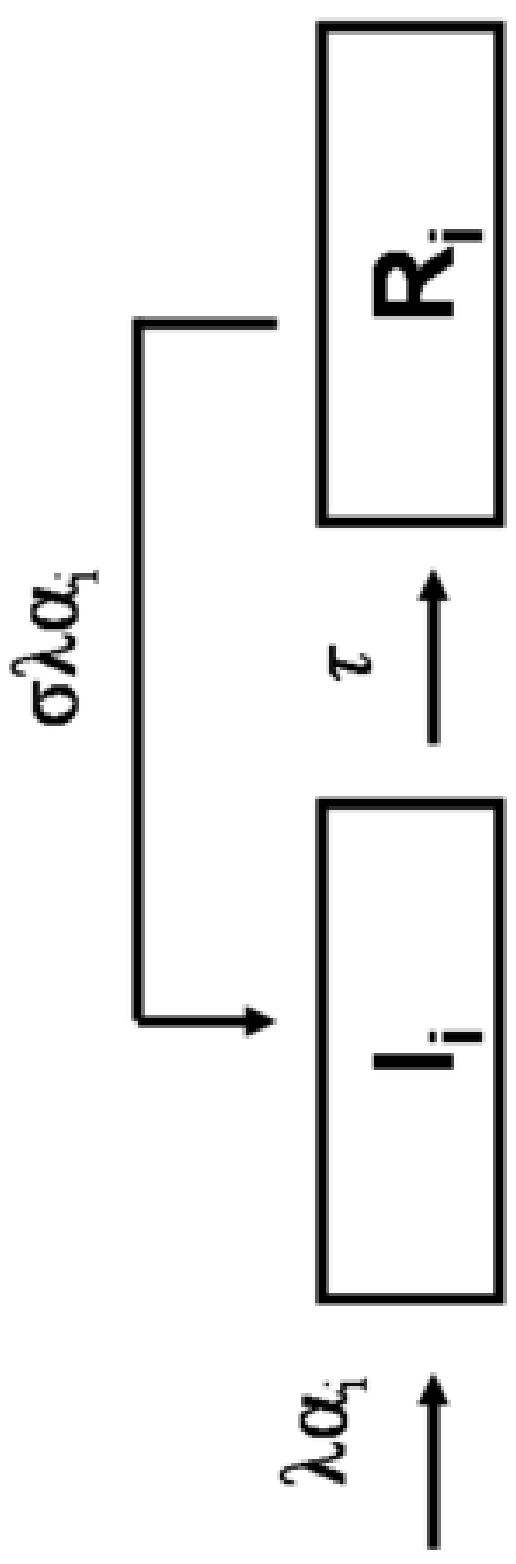

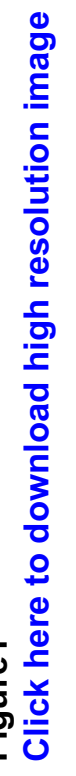
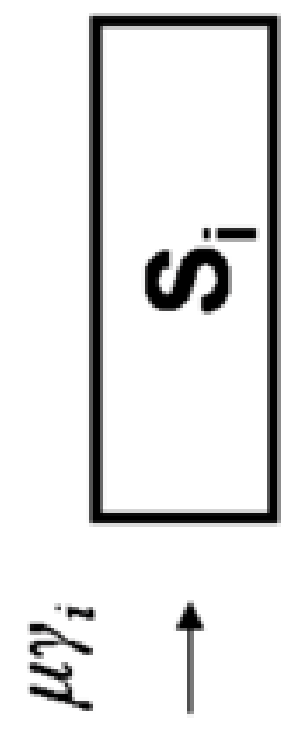

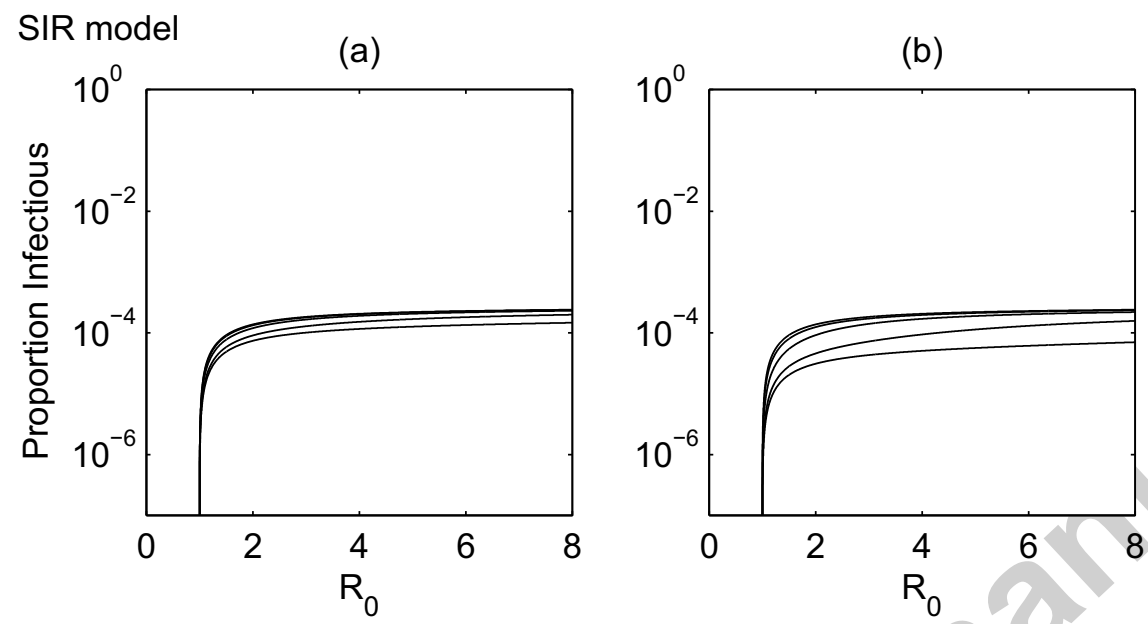

(c)
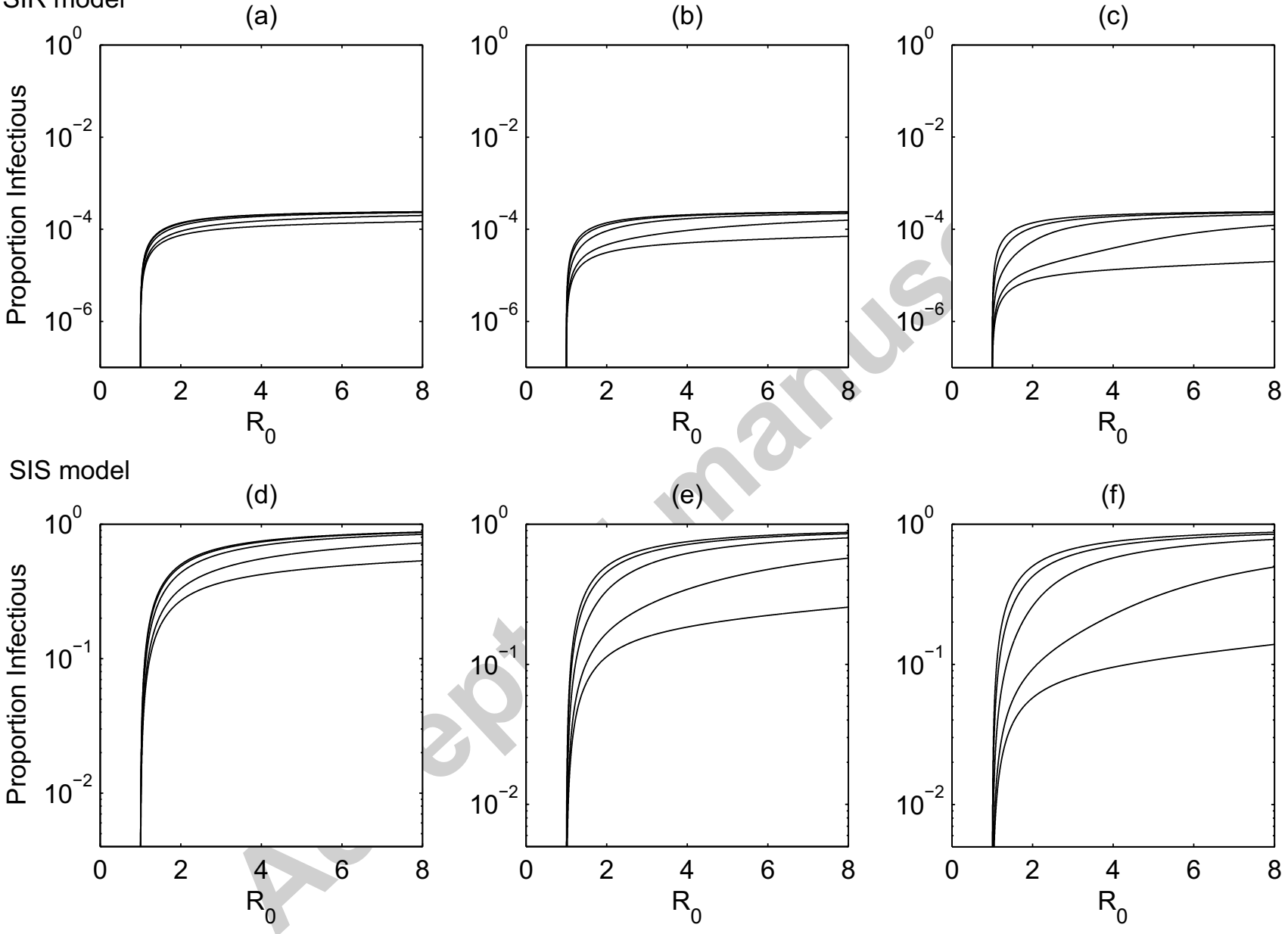
(a)

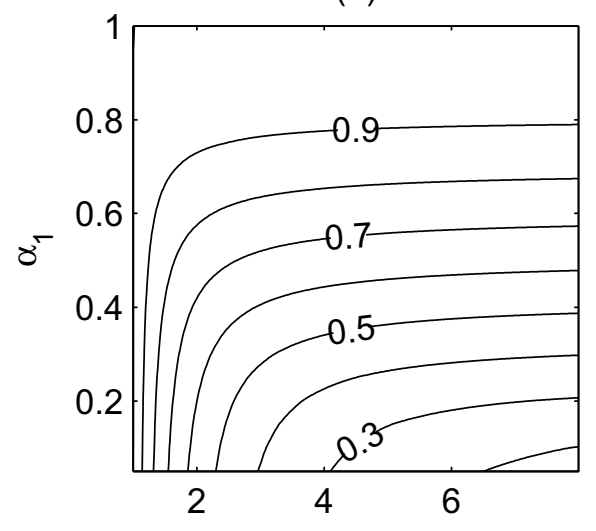

(c)

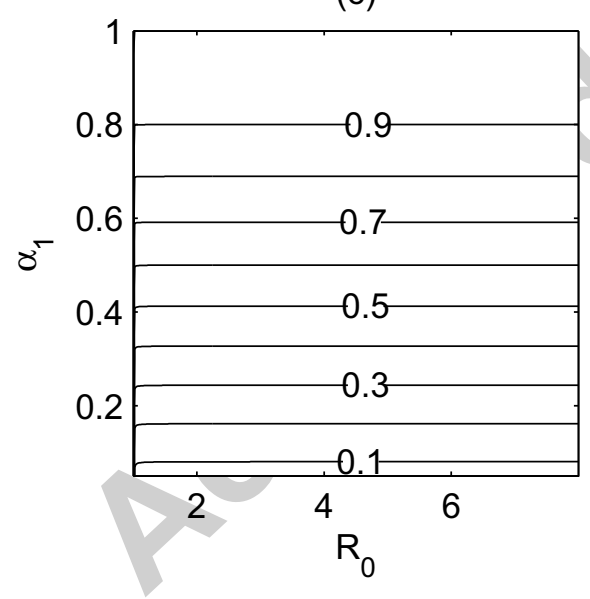

(b)

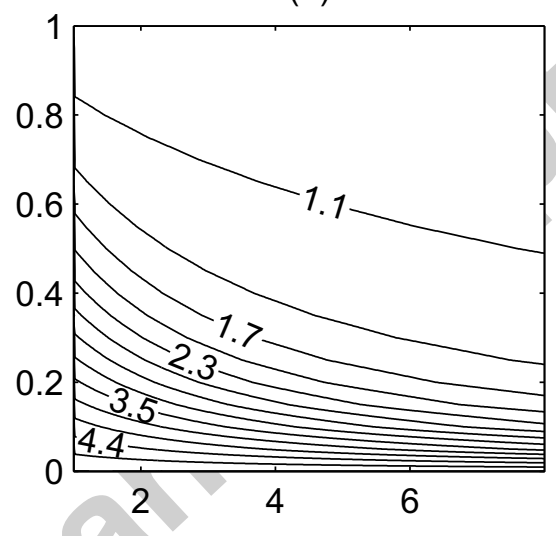

(d)

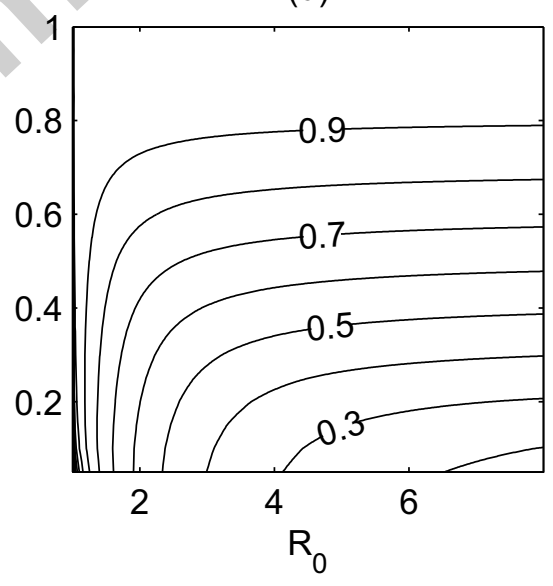


(a)

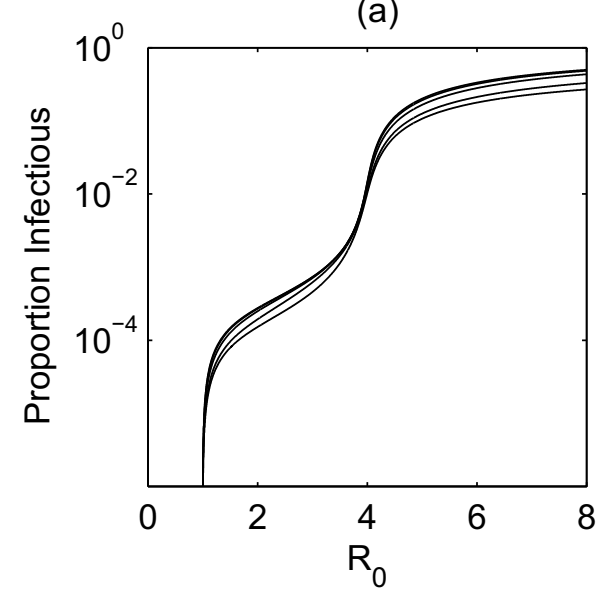

(b)

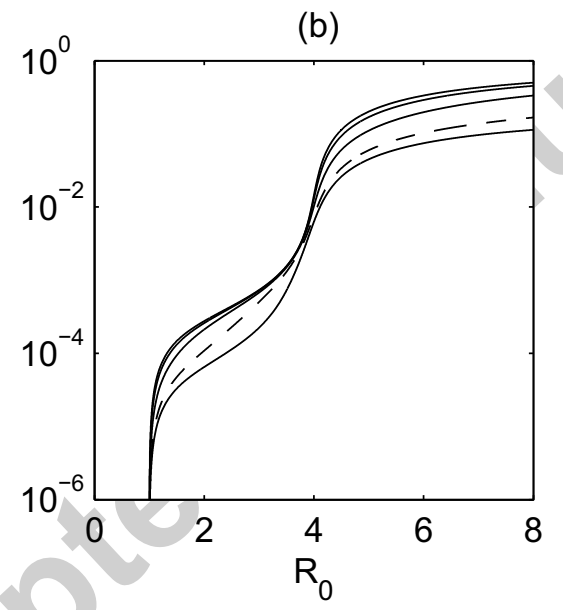

(c)

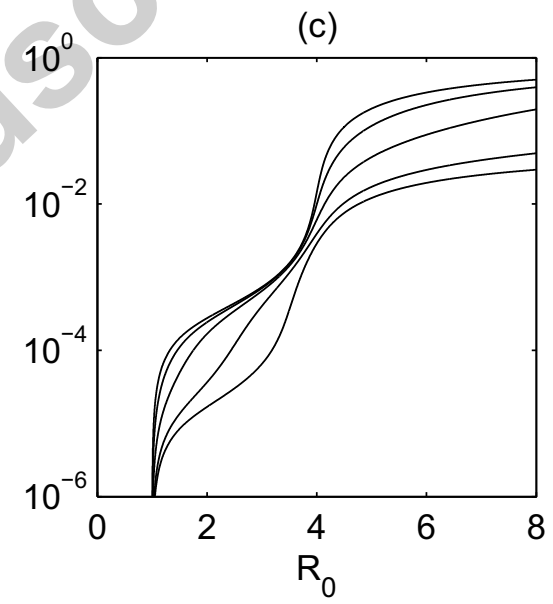


Figure5

Click here to download 4. Figure: Figure5.eps CCEPTED MANUSCRIPT

(a)

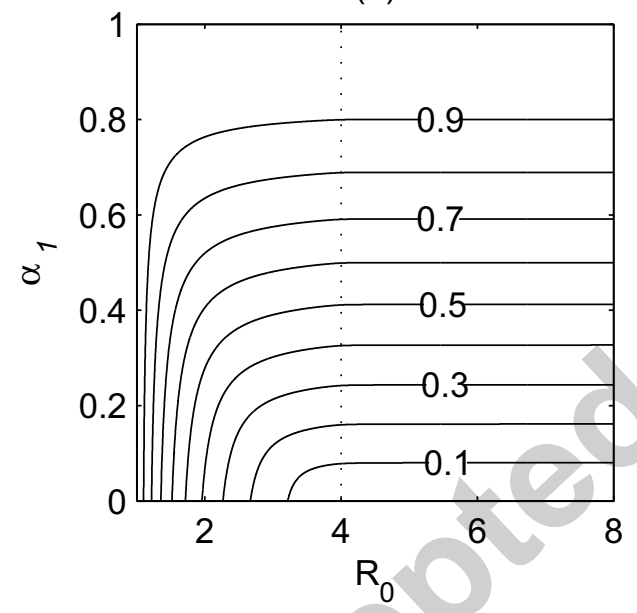

(b)

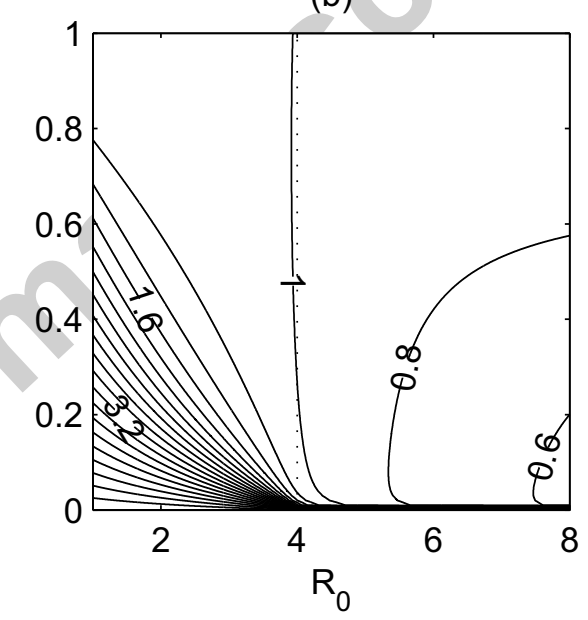


Figure6
Click he

Click here to download 4. Figure: Figure6.eps CCEPTED MANUSCRIPT

(a)

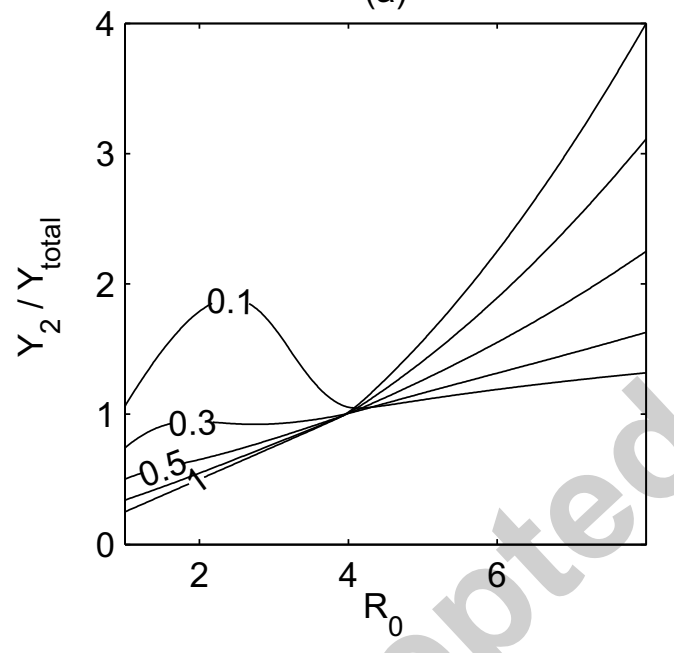

(b)

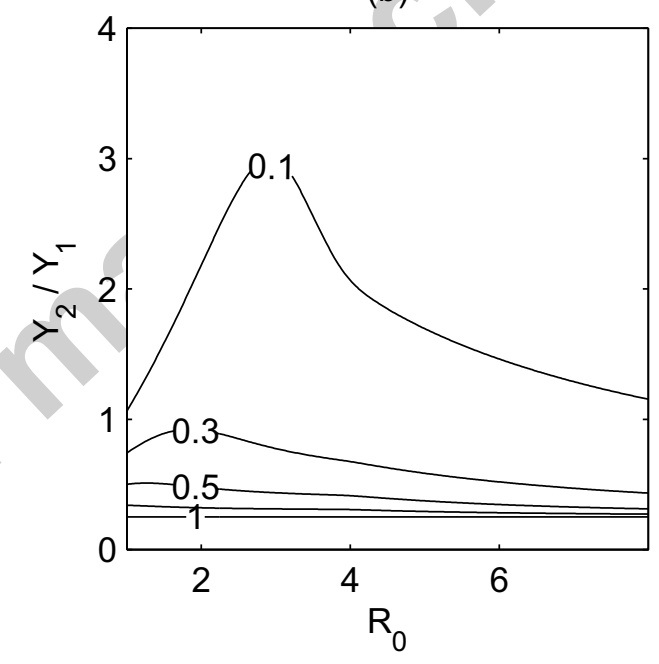


Figure7
Click he

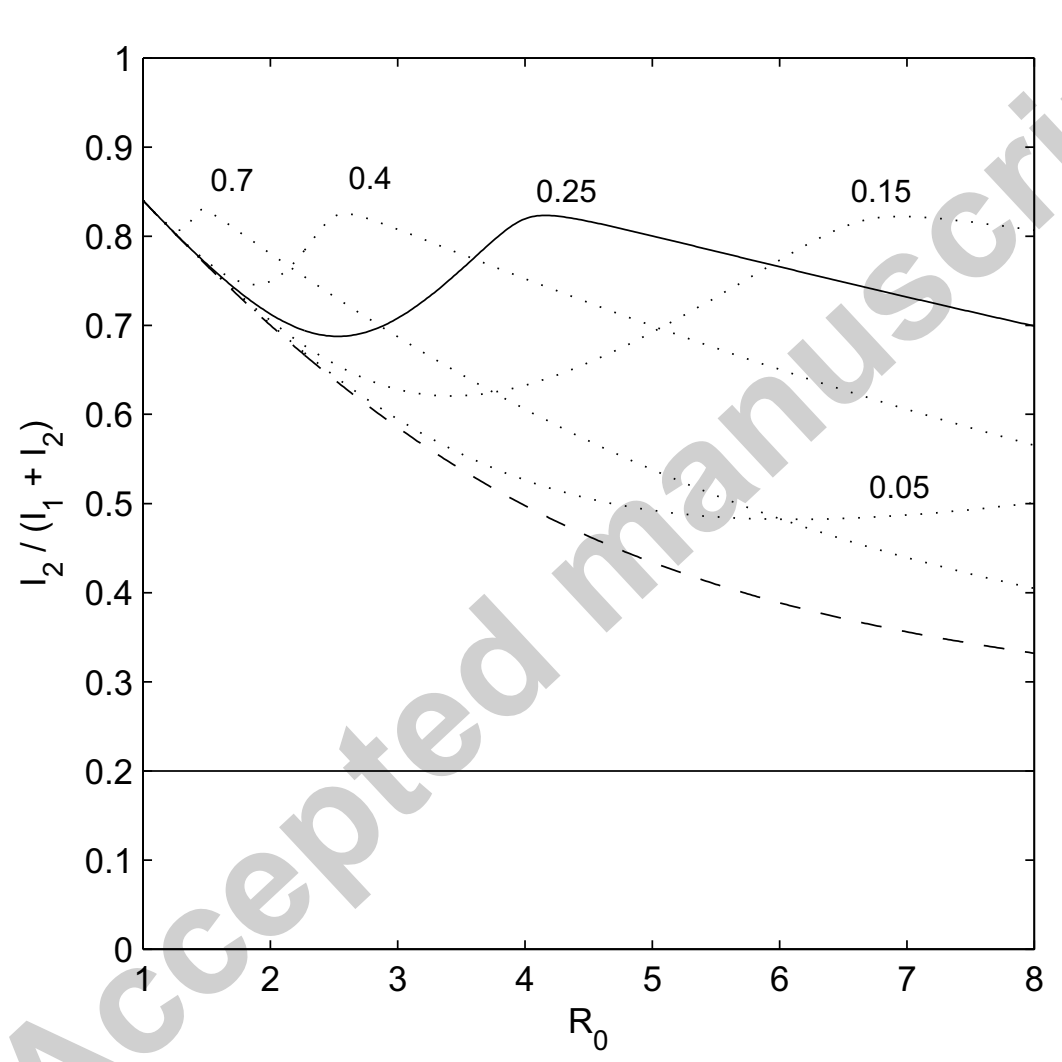

Click here to download 4. Figure: Figure7.eps CCEPTED MANUSCRIPT 
Figure8

(a)

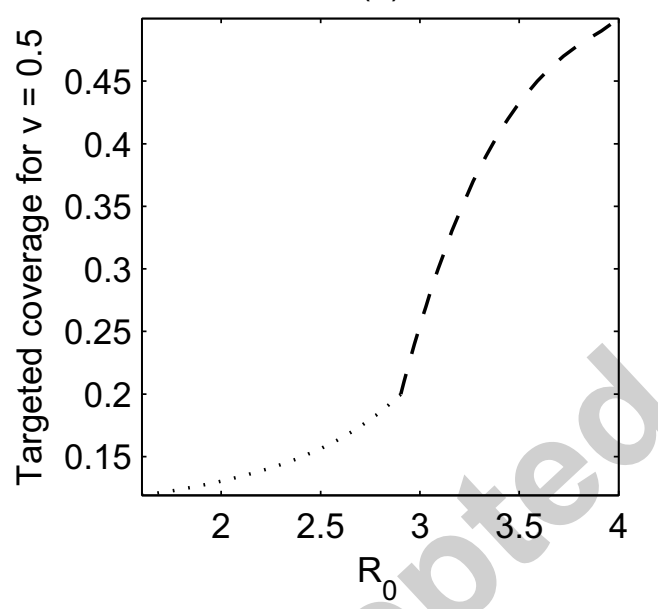

(b)

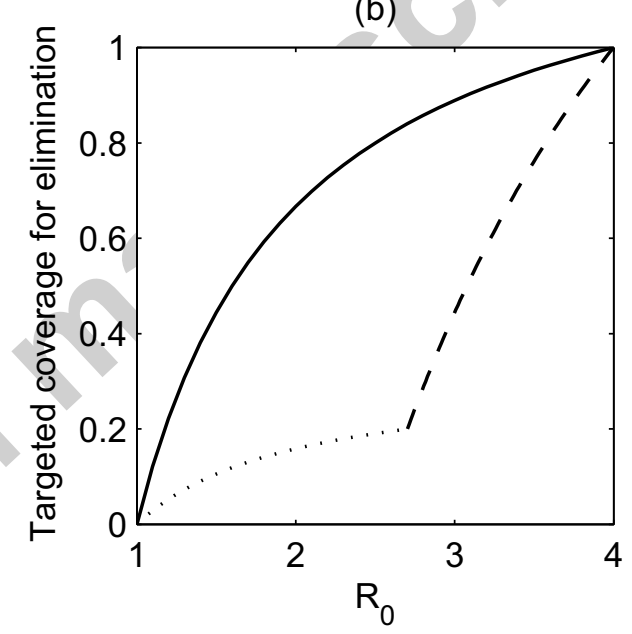


Table 1: about here

\begin{tabular}{cll}
\hline symbol & definition & value \\
\hline$\beta$ & transmission coefficient & variable \\
$\sigma$ & factor reducing the risk of infection as a result of acquired & $0,1 \mathrm{or}$ \\
& immunity to a previous infection & 0.25 \\
$\mu$ & death and birth rate & $1 / 70 \mathrm{yr}^{-1}$ \\
$\tau$ & rate of recovery & $52 \mathrm{yr}^{-1}$ \\
\hline$\gamma_{i}$ & relative size of each frailty group & variable \\
$\alpha_{i}$ & relative risk of infection of each frailty group & variable \\
\hline
\end{tabular}

